\title{
Dynamic Airline Scheduling: An Analysis of the Potentials of Refleeting and Retiming
}

\author{
Warburg, Valdemar; Hansen, Troels G.; Larsen, Allan; Norman, Hans; Andersson, Erik
}

Published in:

Journal of Air Transport Management

Link to article, DOI:

10.1016/j.jairtraman.2008.03.004

Publication date:

2008

Link back to DTU Orbit

Citation (APA):

Warburg, V., Hansen, T. G., Larsen, A., Norman, H., \& Andersson, E. (2008). Dynamic Airline Scheduling: An Analysis of the Potentials of Refleeting and Retiming. Journal of Air Transport Management, 14(4), 163-167. https://doi.org/10.1016/j.jairtraman.2008.03.004

\section{General rights}

Copyright and moral rights for the publications made accessible in the public portal are retained by the authors and/or other copyright owners and it is a condition of accessing publications that users recognise and abide by the legal requirements associated with these rights.

- Users may download and print one copy of any publication from the public portal for the purpose of private study or research.

- You may not further distribute the material or use it for any profit-making activity or commercial gain

- You may freely distribute the URL identifying the publication in the public portal 


\title{
Dynamic Airline Scheduling: An Analysis of the Potentials of Refleeting and Retiming
}

\author{
Valdemar Warburg* \\ Troels Gotsæd Hansen* \\ Allan Larsen (corresponding) ${ }^{*}$ \\ Hans Norman** \\ Erik Andersson*** \\ *DTU Transport \\ Technical University of Denmark \\ Bygningstorvet 1 \\ 2800 Lyngby \\ Denmark \\ ala@transport.dtu.dk \\ **Scandinavian Airlines Sweden \\ Frösundaviks Allé 1 \\ 19587 Stockholm \\ Sweden \\ ***Jeppesen Systems AB \\ Odinsgatan 9 \\ 41103 Gothenburg \\ Sweden
}

\begin{abstract}
We present a Dynamic Airline Scheduling (DAS) technique which is able to change departure times and reassign aircraft types during the booking process to meet fluctuating passenger demands. The procedure is tested on several different days before departure, resulting in a significant profit increase for a major European airline. The results also indicate that applying DAS close to departure yields the largest potential.
\end{abstract}

\section{Keywords}

Schedule optimization, dynamic airline scheduling, retiming, refleeting, passenger forecast.

\section{Introduction}

The scheduling process at major airlines starts well in advance of the actual days of departure, typically one year. The planning and construction of airline schedules are 
evidently based on crude and rather uncertain estimates of the passenger demand on each of the flights in the entire network serviced by the airline. During the booking process, the quality of the passenger demand forecast will increase until the day of departure. In most airlines short-time forecasts of passenger demands are also used for optimizing the utilization of the fleet of aircraft, called refleeting, which means re-allocating the originally assigned types of aircraft to alternative legs in the network.

Dynamic Airline Scheduling, in the following abbreviated DAS is the concept of simultaneously employing refleeting and retiming to the schedule during the booking process in order to adapt the schedule to updated demand forecasts. Refleeting is the idea of adjusting capacity on each leg by reassigning aircraft types to meet actual demands. Retiming is the idea of changing the departure times slightly (e.g. $\pm 15 \mathrm{~min}$ ) based on updated passenger forecasts to accommodate more passenger itineraries or to increase the possibilities of refleeting. Thus, the overall objective of DAS is to increase passenger revenue and reduce cost of flying and thereby increasing the gross profit.

Refleeting is already used by many airlines, while retiming is a new concept and, to the best of our knowledge, only tested by American Airlines. In the academic literature, the concept has only been thoroughly treated by Jiang (2006), but has been discussed sporadically by Etschmaier and Mathaisel (1985), among others.

The work presented in this paper is primarily based on the work of Jiang (2006), but we expand it considerably, in particular by using real data from a more complex network. The concept of Dynamic Airline Scheduling is tested at different days before departure and Jiang's (2006) code is extended to model the problem as real-life as possible. We improve the passenger assignment part of the model significantly by using passenger behavioral results from Adler et al. (2005) and Warburg et al. (2006). Actual data from Scandinavian Airlines (SAS) is used which includes: Real costs, schedules, passenger bookings, fleet information, and passenger forecasts for 26 different days before departure for all 7 days in a chosen focus week in September, 2006. Tests are performed on the entire schedule of SAS, and show an increase in gross profit of $1 \%$ by performing DAS close to departure. Finally, we provide an analysis of the impact of broken crew rules and suggest how this can be minimized.

\subsection{Previous Studies}

In the literature, much attention has been devoted to each of the two elements in DAS: Fleet assignment and aircraft scheduling. Within fleet assignment, we distinguish between the static and dynamic environments. In the static environment, fleet assignment is done well before departure and only once. In the dynamic environment, the fleet assignment has already been completed and the focus is on adjusting the fleet assignment to cater for new information such as updates in demand estimates.

Studies within the static environment include Clarke et al. (1996), who treat the problem of static fleet assignment while taking some maintenance and crew considerations into account. Rexing et al. (2000) treat the problem of fleet assignment with time windows, while Barnhart et al. (2002) present the passenger mix model (i.e. how to assign the 
correct mix of passengers) and integrate it with a basic fleet assignment model to obtain an itinerary-based fleet assignment model. The passenger mix model presented in the present paper is a variation of the passenger mix model presented in Barnhart et al. (2002) that ignore the recapture rates, because they are hard to estimate. For more details on recapture rates, see Barnhart et al. (2002) and Jiang (2006). Yan et al. (2007) develop a flight scheduling model that applies both fleet assignment and flight scheduling under variable market shares estimated by choice models.

Studies within the dynamic environment include Berge and Hopperstad (1993), who introduced the idea of refleeting based on updated passenger forecasts. The DAS model is an extension of this work and introduces the dynamic scheduling dimension. Talluri (1996) treats the problem of refleeting aircraft for both operational purposes and to recover from disruptions. Jarrah et al. (2000) consider a refleeting model for incremental modifications of planned fleet assignments. These modifications are caused by operational issues, while the idea of DAS is to enhance the schedule based on updated passenger forecasts.

Conclusively, little attention has been paid to the problem of adjusting the schedule and adjusting the fleet assignment based on updated passenger forecasts at a later stage in the planning process. This paper addresses exactly these issues.

The paper is organized as follows: Section 2 presents the DAS model framework discussing the proposed improvements. Section 3 contains results of different scenario runs, as well as well as a discussion of how crew schedules are affected by DAS. Finally, we provide our concluding remarks in section 4.

\section{Model Framework}

The two models used in the current study are the Passenger Mix Model (PMM) and the Dynamic Airline Scheduling model (DAS). For a mathematical description of the models, see Jiang (2006). First, two cases will be defined:

1) A static case, in which passengers are assigned to the original schedule without reoptimizing. The static case uses only PMM.

2) A dynamic case, in which the schedule is reoptimized at a chosen reoptimization point. The dynamic case uses both PMM and DAS.

Additionally, two intervals are defined before the day of departure, separated by a reoptimization point: Period 1 lasts from the moment the booking period begins until the reoptimization point, and Period 2 lasts from the reoptimization point until the day of departure.

Actually booked passengers and an original schedule is the input data for period 1 in the static case. The PMM model then assigns the booked passengers to the schedule. Input data for period 2 includes passenger demand, the original schedule, and a list of occupied seats from period 1 . The latter is to prevent passengers from being assigned to already booked seats. 
In the dynamic case, the DAS model is used at the reoptimization point, as shown in Figure 1. Period 1 is identical to the static case with the same input data. However, at the reoptimization point, the input data is the period 2 forecast, captured at that particular point. Additionally, the number of overnighting aircraft at each city ensures that the reoptimized schedule does not conflict with the next day's schedule. The list of protected connections (which are the transfers that the passengers in period 1 have been assigned) ensures that a flight will not be retimed in such a way that a period 1 passenger cannot make his connecting flight. The DAS model retimes the different flight departures by previously defined minute intervals (in this case \pm 10 and \pm 15 minutes) and refleets aircraft types between different flight legs, so that the schedule is optimized based on the available period 2 forecast at that time. In period 2, PMM assigns the passenger demand for period 2 to the newly reoptimized schedule with retimed flights and refleeted aircraft. As in the static case, it uses the list of occupied seats from period 1 to prevent passengers from being assigned to booked seats.

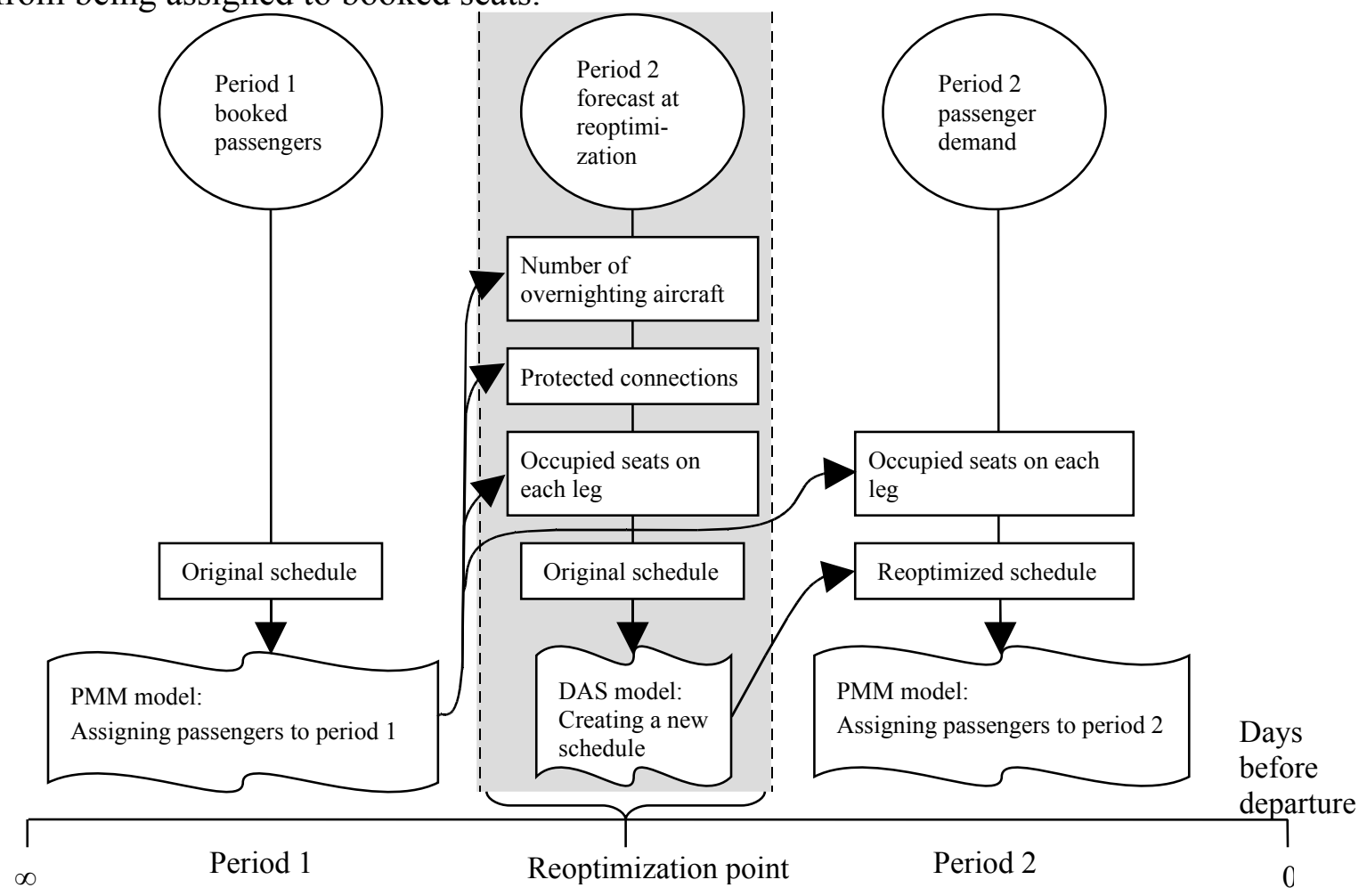

Figure 1. Model framework for the dynamic case with a reoptimization point, in which the Dynamic Airline Scheduling model is used. In the static case, nothing is done at the reoptimization point.

\subsection{Improvements of the Choice Model}

One of the most critical factors in the Passenger Mix Model and the Dynamic Airline Scheduling model is the assignment of passengers. If an itinerary fulfills a passenger's departure and arrival airports of choice, departure period and desired number of stops, the passenger will choose the itinerary and pay the offered fare with $100 \%$ certainty. 
However, this procedure presents a number of uncertainties. Several other characteristics, such as flight time, on-time performance, airport choice, and aircraft type, contribute to determining a choice window.

In this study, the choice modeling of departure periods is improved by adjusting them more adequately to the market of SAS. While Jiang (2006) only defines four different departure periods (morning, afternoon, evening, and night) for all passengers, we define departure periods around each departure time in the input data, thus adapting the preferred departure period for each passenger in 30 minute intervals.

Jiang (2006) defines broad departure periods varying between 7 and 9 hours. This is a rough generalization of passengers' departure choice, and one should distinguish between business and non-business passengers. Parker and Walker (2005) find that business passengers are twice as sensitive to departure time difference as non-business passengers. Adler et al. (2005) experience that business passengers are six times as sensitive to arrival time difference as non-business passengers (arrival time being comparable to departure time in an origin-destination environment), and Warburg et al. (2006) find that the difference is three-fold. Based on these studies, $a \pm 1$ hour departure period is assigned to Business class passengers and $\mathrm{a} \pm 3$ hours departure period to Economy class passengers. Note that this is a generalization, given that not all business passengers fly Business class and vice versa.

\subsection{Improvement of Cost Modeling}

Correct representation of fleet costs is essential to achieve a reliable model. Jiang (2006) models the costs as a fixed cost and a variable cost, while the current study has enhanced the modeling of variable cost and introduced two new cost elements - traffic variable cost and extra fuel cost.

The fixed cost is the cost of using an airplane of a certain type on a particular day. Jiang (2006) assumes that this money is saved, if the model is able to decrease the number of aircraft used. However, according to SAS, it is very unlikely to find another use of the airplane just a few weeks in advance, which is why the fixed cost has been set to zero in the current study.

The representation of variable cost is improved in the current study by distinguishing between two elements: 1) cost of taking off with an aircraft of a given type, and 2) the cost of operating the airplane one block hour. This takes into consideration the high cost of flying short-haul flights as compared to long-haul flights per distance flown.

The traffic variable cost represent the cost of flying a passenger on a specific leg, independent of which aircraft is used. The cost includes food, beverages, newspapers and all other in-flight costs directly associated with the passengers. The extra fuel cost is a rough estimate of how extra passengers affect fuel usage, and must be multiplied by the number of passengers and number of block hours. 


\section{Results}

The program is implemented in C++ using the ILOG CPLEX 9.0 software package with its MIP optimizer. All tests were performed on an Intel Pentium 4, $2.80 \mathrm{GHz}$ with $2 \mathrm{~GB}$ of RAM.

PMM is solved to optimality in approximately one second. DAS, however, is much harder to solve. Each run has been stopped after four hours of iterations, as the solution only improves marginally after this time.

The forecast is available at 26 specific days before departure, in short $d b d$ 's. The booking pattern during the time period from $d b d 200$ (which is the earliest $d b d$ ) until $d b d 0$ (the day of departure) is examined in order to determine appropriate optimization points. The optimization points $d b d 79,49,34,24,15,11,7$ are chosen based on a criterion of evenly spread of bookings and a criterion of including extreme (very early and very late) $d b d$ 's.

Table 2 shows the differences in gross profit between the static case and the dynamic case for each weekday for each day before departure $(d b d)$.

\begin{tabular}{|l|c|c|c|c|c|c|c|c|}
\hline \multicolumn{7}{|c|}{ Gross profit differences between static case and dynamic case } \\
\hline \multicolumn{1}{|c|}{$\boldsymbol{d b d}$} & $\mathbf{1}$ & $\mathbf{7}$ & $\mathbf{1 1}$ & $\mathbf{1 5}$ & $\mathbf{2 4}$ & $\mathbf{3 4}$ & $\mathbf{4 9}$ & \multicolumn{1}{|c|}{$\mathbf{7 9}$} \\
\hline Day & & & & & & & & \\
\hline Tonday & $1.05 \%$ & $1.23 \%$ & $1.02 \%$ & $1.13 \%$ & $0.16 \%$ & $-0.11 \%$ & $-0.11 \%$ & $-0.09 \%$ \\
\hline Wednay & $0.92 \%$ & $1.14 \%$ & $1.10 \%$ & $1.12 \%$ & $0.99 \%$ & $0.90 \%$ & $0.90 \%$ & $0.51 \%$ \\
\hline Thursday & $0.00 \%$ & $1.01 \%$ & $1.13 \%$ & $0.96 \%$ & $0.82 \%$ & $0.32 \%$ & $0.07 \%$ & $0.10 \%$ \\
\hline Friday & $0.00 \%$ & $1.28 \%$ & $0.53 \%$ & $0.81 \%$ & $0.58 \%$ & $0.71 \%$ & $0.66 \%$ & $0.63 \%$ \\
\hline Saturday & $0.92 \%$ & $1.14 \%$ & $0.72 \%$ & $0.64 \%$ & $0.54 \%$ & $0.49 \%$ & $0.49 \%$ & $0.70 \%$ \\
\hline Sunday & $1.63 \%$ & $1.17 \%$ & $0.90 \%$ & $0.41 \%$ & $-0.13 \%$ & $0.29 \%$ & $0.04 \%$ & $-0.84 \%$ \\
\hline
\end{tabular}

Table 1. Differences in gross profit for each weekday for each optimization point.

The profit differences vary from $-0.8 \%$ to $1.6 \%$. In general, the benefits increase as the departure day approaches. This most likely due to increase in the forecast quality.

There are a few negative profit differences, for Monday, Saturday, and Sunday, primarily for the early $d b d$ 's. Evidently, the DAS model has in these cases developed a reoptimized schedule that is unsuitable for the rest of the assigned demand so that the static case yields a higher gross profit than the dynamic case. The likely reason for this is that the forecast is of a bad quality at those early $d b d$ 's on those particular days. Still, for most weekdays there is a profit increase even at the early $d b d$ 's. Note also that for Wednesday and Thursday ( $d b d 1)$, the model could not find a better schedule solution than the existing schedule, resulting in a profit difference of zero.

On average, revenue increase constitutes $20 \%$ of the total profit increase, and cost reduction constitutes $80 \%$. This indicates that the major part of the benefits is obtained through refleeting, or through the combination of retiming and refleeting. Retiming in itself does not contribute much. This issue is investigated further in section 3.1. 
Jiang (2006) experiences gross profit increases from 1.97\% to 4.91\%, when using historical average as forecast, which is likely due to the fact that the airline studied is almost completely hub-and-spoke based and has more than twice the amount of traffic at the major hub, compared to SAS' major hub, which leaves more potential for DAS. Furthermore, Jiang (2006) uses very broad departure periods, and depeaks the airline's schedule from an originally peaked schedule. A model-based, optimally depeaked schedule is likely to form a better basis for DAS.

In the following, a number of sensitivity scenarios are presented with focus on the two $d b d$ 's 49 and 15. These two $d b d$ 's represent two specific time points in the planning process. At $d b d 49$, crew paring, rostering, and tail assignment have not been done. Thus, DAS can be performed without causing difficulties for any other departments at SAS. Contrary, at $d b d 15$, the crew plans have already been made, and therefore it is necessary to analyze the impact of DAS on crew plans. This analysis is performed in section 3.4.

\subsection{Contributions from Retiming and Refleeting}

Tests on the Tuesday schedule are performed to investigate the impact of retiming and refleeting, respectively on the network of SAS. The results show that the impact of only doing retiming is insignificant for all $d b d$ 's, while the impact of refleeting is much larger. Most significant is the fact that applying both retiming and refleeting results in an average $60 \%$ increase in gross profit gain. For example, for $d b d 7$, the result of retiming is only $0.02 \%$ and for refleeting it is $0.70 \%$. However, for both retiming and refleeting the profit increase is $1.14 \%$. The presence of this synergy is reinforced by the fact that approximately 50 (one third) of the retimed legs are also refleeted. The synergy occurs when retiming is performed in order to achieve refleeting or vice versa.

This is contrary to Jiang (2006), who shows that the contribution of only doing retiming is larger than the contribution of only doing refleeting. However, this is likely due to the fact that the airline studied on average has 4 arrivals and 4 departures every 10 minute to and from the main hub during the busiest hours from 8 AM to 8 PM. SAS, however, has on average 2 arrivals and 2 departures at their major hub, Copenhagen $(\mathrm{CPH})$ and a little less at their two minor hubs, Stockholm (ARN) and Oslo (OSL). So even though SAS actually has more flights than the airline studied by Jiang, the departures are split out on three hubs, reducing the potential of only retiming. More traffic at the main hub provides more connection possibilities when retiming the flights and therefore a larger potential for only retiming.

\subsection{Different Retiming Values}

Alternative combinations of retiming values are investigated for Tuesday for $d b d 15$ and 49. These include: \pm 5 and 15 min., \pm 10 and 15 min., \pm 15 and 20 min., and \pm 15 and 30 $\mathrm{min}$. The results show that there is significant variation between the retiming value combinations, depending on whether reoptimization is done at $d b d 15$ (resulting in a $0.8 \%-1.1 \%$ gross profit increase) or at $d b d 49$ (resulting in a $0.2 \%-0.6 \%$ gross profit increase) . In fact, the combination \pm 15 and \pm 20 minutes generates the highest profit increases when reoptimizing at $d b d 15$, but the second-lowest, when reoptimizing at $d b d$ 
49. The opposite is the case for the combination \pm 5 and \pm 15 min. For both $d b d$ 's, the combination \pm 15 and \pm 30 min. yields the lowest profit increases. This could indicate that retiming values should be kept below 30 minutes to obtain the largest benefits. Our standard retiming values ( $\pm 10 \mathrm{~min}, \pm 15 \mathrm{~min}$ ) are in the average range for both $d b d$ 's.

One could argue that the willingness-to-pay will decrease (and thereby the demand), if the passengers' sensitivity to short scheduling changes is high. However, airlines already make 15 minute changes one or few days before departure due to operational issues, and notify passengers by phone or via text messages. Additionally, it is quite common that flights are 15 minute late or more, and thus, this extra margin will usually be included in the passengers' schedule. However, it may become an increasing problem if the retiming values are $\pm 30 \mathrm{~min}$.

\subsection{Variation of Departure Periods}

The definition of departure periods is subject to much uncertainty, particularly because passenger choice is such a complex field. Hence, in order to test the sensitivity of the departure periods, two alternative departure period scenarios are defined. This is achieved by doubling the periods (meaning \pm 6 hours for Economy passengers and \pm 2 hours for Business passengers) and halving the periods (meaning \pm 1.5 minutes hours for Economy passengers and \pm 30 minutes for Business passengers). The Double departure periods yielded 29\% higher gross profit increases than the Standard departure period, while the Half departure periods yielded 36\% lower gross profit increases. The tests were run for a Tuesday schedule on $d b d 15$.

The results indicate that the model exploits the flexibility of the passengers by assigning them to less crowded aircraft. This emphasizes the importance of having the most correct estimations of departure periods available. However, the variations between Double and Half departure periods are not significant enough to sow doubts about the original results, so the Half and Double scenarios can be treated as upper and lower bounds.

\subsection{Crew Considerations}

As the results reveal, the largest potential for DAS is after $d b d 15$, which is, in most airlines, after crew plans have been distributed. Therefore, it is necessary to investigate how many crew and maintenance constraints that will be broken. According to Berge and Hopperstad (1993), it is usually not a problem to reschedule maintenance after some refleetings have been made. Also, maintenance is often rescheduled quite late by many airlines today. Therefore, this paper will not treat maintenance analysis, but rather focus on the more critical crew issue.

An analysis has been performed using crew scheduling software from Jeppesen Systems. We consider flight deck pairings on the Tuesday in week 37 for $d b d 15$ for SAS Braathens (the Norwegian branch of SAS), and the optimization software Carmen Crew Pairing reveals that 23 out of 108 crew pairings on that day are infeasible (21\%). SAS Braathens has on this day 399 legs, of which 162 legs were changed by DAS (refleeted 
and/or retimed). Almost all infeasibilities are due to the rule that the crew must have a certain minimum connection time between two legs, which varies across airports.

One method of reducing infeasible pairings is to make the pairings less sensitive to time and aircraft changes. This can be achieved by increasing the connection time when there is a flight change, or by minimizing the total number of flight changes. We have tested the method by imposing a standard 50-minute minimum connection time for all crew at any connection. When comparing with the normal smaller connection time, only 10 pairings become infeasible. So by introducing just a little more slack in the connection time, the number of infeasible parings is reduced from 23 to only 10. Additionally, this change only imposes a minor rise in productivity costs. This indicates that it may be possible to reduce the number of infeasible pairings by investigating the issue with connection times. Another option is to introduce new constraints in DAS that avoid breaking the tight crew connections, which, however, would limit the benefit of DAS.

A more long-term solution is to integrate crew planning with DAS, thus allowing crew, schedule and fleet changes up until a week before departure. However, this would pose great organizational challenges for established airlines and would also conflict with existing union agreements.

\subsection{Benefits for different airline types}

As the results from Jiang (2006) show, a hub-and-spoke airline with a single-hub network is likely to benefit the most from applying DAS to its network as the number of refleeting/retiming combinations increase substantially, when the majority of the traffic travels through a single hub. SAS has three major hubs and will therefore potentially benefit less than a single-hub airline. Essentially, single-hub airlines such as Air France (with its Paris hub), AeroMexico (with its single Mexico City hub) and Delta (with its Atlanta hub, although it has additional hubs) are expected to benefit most from DAS. Additionally, airlines that have high frequencies on the same routes are expected to have a higher benefit from retiming, since numerous flight combinations with the same destination are then offered to the passenger.

\section{Conclusion}

Dynamic Airline Scheduling is performed on eight selected days before departure with substantial increase in gross profit as a result. The impact of retiming diminishes, when no refleeting is done, whereas performing both retiming and refleeting together results in substantially higher benefits due to a synergy effect. Some crew rules are broken, mostly because of insufficient crew connection times, but the analysis shows that the number of broken rules can be decreased considerably.

\section{Acknowledgements}

The authors would like to express their thanks to Dr. Hai Jiang and Professor Cynthia Barnhart at MIT for providing us access to the Dynamic Airline Scheduling code, which is the basis for the current research. 


\section{References}

Adler, T., Falzarano, C. S., and Spitz, G., 2005. Modeling Service Trade-offs in Air Itinerary Choices. Transportation Research Record, 1915: pp. 20-26.

Barnhart, C., Kniker, T. S., and Lohatepanont, M., 2002. Itinerary-Based Airline Fleet Assignment. Transportation Science, 36(2): pp. 199-217.

Belanger, N., Desaulniers, G., Soumis, F., and Desrosiers, J., 2006. Periodic Airline Fleet Assignment with Time Windows, Spacing Constraints, and Time Dependent Revenues. European Journal of Operations Research, 175: pp. 1754-1766.

Berge, M. E. and Hopperstad, C. A., 1993. Demand Driven Dispatch: A Method for Dynamic Aircraft Capacity Assignment, Models and Algorithms. Operations Research, 41(1): pp. 153-168.

Clarke, L. W., Hane, C.A., Johnson, E. L., and Nemhauser, G. L., 1996. Maintenance and Crew Considerations in Fleet Assignment. Transportation Science, 30(3): pp. 249260.

Desaulniers, G., Desrosiers, J., Dumas, Y., Solomon, M. M., and Soumis, F., 1997. Daily Aircraft Routing and Scheduling. Management Science, 43(6): pp. 841-855.

Etschmaier, M. M. and Mathaisel, D. F. X., 1985. Airline Scheduling: An Overview. Transportation Science, 19(2): pp. 127-138.

Jarrah, A., Goodstein, J., and Narasimhan, R., 2000. An Efficient Airline Re-Fleeting Model for the Incremental Modification of Planned Fleet Assignments. Transportation Science, 34(4): pp. 349-363.

Jiang, H., 2006. Dynamic Airline Scheduling and Robust Airline Schedule De-Peaking. Ph.D. thesis, Massachusetts Institute of Technology.

Parker, R. A. and Walker, J., 2006. Estimating Utility of Time-of-Day Demand for Airline Schedules Using Mixed Logit Model. Presented at Transportation Research Board Annual Meeting 2006, Washington DC.

Rexing, B., Barnhart, C., Kniker, T., Jarrah, A., and Krishnamurthy, N., 2000. Airline Fleet Assignment with Time Windows. Transportation Science, 34(1): pp. 1-20.

Talluri, K. T., 1996. Swapping Applications in a Daily Airline Fleet Assignment. Transportation Science, 30(3): pp. 237-248.

Warburg, V., Bhat, C. R, and Adler, T., 2006. Modeling Demographic and Unobserved Heterogeneity in Air Passengers' Sensitivity to Itinerary Choice. Transportation Research Record, 1951: pp. 7-16.

Yan, S., Tang, C.-H., and Lee, M.-C., 2007. A Flight Scheduling Model for Taiwan Airlines under Market Competitions. Omega, 35: pp. 61-74. 